\title{
A RETROSPECTIVE STUDY OF THE IMPACT OF RADIATION FRACTIONATION ON PAINFUL BONE METASTASIS
}

\author{
Suresh Kumar K1, Flowerlit Thomas², Ajith Nair ${ }^{3}$, Mintu Mathew ${ }^{4}$, Snigdha P. P5 \\ ${ }_{1}^{1}$ Additional Professor, Department of Radiotherapy, MCH, Kottayam, Kerala, India. \\ ${ }^{2}$ Assistant Professor, Department of Radiotherapy, MCH, Kottayam, Kerala, India. \\ ${ }^{3}$ Lecturer, Department of Radiotherapy, MCH, Kottayam, Kerala, India. \\ ${ }^{4}$ Lecturer, Department of Radiotherapy, MCH, Kottayam, Kerala, India. \\ ${ }_{5}^{5}$ Lecturer, Department of Radiotherapy, MCH, Kottayam, Kerala, India.
}

\section{BACKGROUND}

ABSTRACT

Bone metastasis and bone pain are most detrimental to the life of cancer patients. There is a significant discrepancy between the physician estimate of pain and the pain level reported by the patient. We wanted to evaluate the effectiveness of radiotherapy in reducing the bone pain and identify the suitable dose fractionation schedule.

\section{METHODS}

This is a retrospective cohort study analysis carried out in patients with bone metastasis who presented to radiotherapy department, MCH Kottayam from August 2010 to January 2015 and were treated with radiotherapy. Data collected from the case records to assess the response to radiotherapy in patients who were treated with either 20GY/5\# or 8GY/1\#, response was assessed periodically with the universal pain assessment tool.

\section{RESULTS}

Even though there was difference in the type of pain controlled by the two radiation fractions, the overall pain score showed no statistically significant difference in the overall pain control between the two arms.

\section{CONCLUSIONS}

$20 \mathrm{~Gy} / 5$ fractions and $8 \mathrm{~Gy} /$ single fraction schedules of radiotherapy were found to be similar in terms of rates of pain relief, narcotic use, and pathologic fracture. In terms of acute control of patient symptoms of bone pain, either of the two schedules may be used without compromising outcome.

HOW TO CITE THIS ARTICLE: Kumar SK, Thomas F, Nair A, et al. A retrospective study of the impact of radiation fractionation on painful bone metastasis. J. Evolution Med. Dent. Sci. 2019;8(13):995-1002, DOI: 10.14260/jemds/2019/222

\section{BACKGROUND}

Bone metastasis and bone pain are most detrimental to quality of life of cancer patients. The incidence of bone metastases varies significantly depending on the primary site, with breast and prostate cancer accounting for up to $70 \%$ of patients with metastatic disease ${ }^{1}$. Other primary sites with a propensity for bone metastases include thyroid, melanoma, and kidney. Some hematologic malignancies including myeloma and lymphoma can also cause significant pain and bone destruction. Solitary bone metastases occur in $<10 \%$ of patients.

Metastases can occur in any bone, though they are more common in sites containing red bone marrow. Approximately $70 \%$ of bone metastases occur in the axial skeleton, with metastasis most frequently occurring in the spine, pelvis, and ribs. The lumbar spine is the single most frequent site of bone metastasis. $^{2}$

'Financial or Other Competing Interest': None.

Submission 29-11-2018, Peer Review 01-03-2019,

Acceptance 08-03-2019, Published 01-04-2019.

Corresponding Author:

Dr. Flowerlit Thomas,

Assistant Professor,

Department of Radiotherapy,

MCH, Kottayam,

Kerala, India.

E-mail: litjino@gmail.com drsureshkumar74@gmail.com

DOI: $10.14260 /$ jemds $/ 2019 / 222$
In the appendicular skeleton, the proximal femurs are the most common site of metastatic disease. Overall survival of bone metastasis depends on the primary site and the presence or absence of visceral metastases. ${ }^{3}$

The most common symptom of bone metastases is slowly progressive, fairly well localized insidious pain may radiate to other areas. The pain may be worse at night. Approximately $1 \%$ of bone metastases lead to pathologic fracture, with annual fracture rates of $5-20 \%{ }^{4}$

The mechanism of pain from bone metastases is not clearly understood. Possible mechanisms include mechanical instability, irritation of periosteal stretch receptors, tumourdirected osteoclast-mediated osteolysis, and tumour cells themselves, or tumour-induced nerve injury, production of nerve growth factor or stimulation of other cytokine receptors. ${ }^{5}$

\section{Prognostic Factors}

1. Histology: median survival of patients with Bone metastases in breast, prostate, and lung cancer are 69, 40 , and 13 weeks, respectively.

2. Presence of only a limited number of bone metastases and absence of visceral metastases is a favourable factor.

3. Good performance status.

4. Absence of co morbidities.

5. Response to treatment especially hormone or chemotherapy. 


\section{Evaluation}

The physical examination is an important step in evaluating a patient with bone metastases. Firm palpation will often elicit the specific area of pain, with point tenderness often pointing directly to the affected area in the bone. It is important to carefully evaluate the entire skeletal system with examination, as intense pain at one site often masks subjective reports of pain at other sites. A thorough neurologic examination is also important, especially in patients with spinal metastases, to carefully evaluate for the possibility of spinal cord, cauda equina, or nerve root compression.

\section{Imaging}

Plain radiographs are typically the most appropriate first imaging study. The primary disadvantage of plain radiographs is that small lesions are rarely seen. Approximately $30 \%$ to $50 \%$ of the bone mineral content must be lost before the lesion will be apparent on x-rays. ${ }^{6}$

Comparative studies have shown PET scans to be more sensitive than Tc-99 m scintigraphy or whole-body MRI scans in detecting bone metastases. ${ }^{7}$

\section{Pain Management}

There is a significant discrepancy between the physician estimate of pain and the pain level reported by the patient. 8 The use of a validated pain scale, such as the Brief Pain Inventory or universal pain assessment tool, gives the patient an opportunity to describe the severity of pain and the interference of pain with function in a manner that can be understood both by the patient and the physician ${ }^{9}$. This also allows for comparisons of pain levels over time, to better assess the effectiveness of treatments.

Pain control can be achieved in the majority of patients using the World Health Organization analgesic ladder. The WHO three-step analgesic ladder provides the most straightforward and universally accepted approach to analgesia. The ladder describes a process for combining nonopioid, opioid, and adjuvant drugs, titrated to meet the individual needs of the patient according to the severity of pain. A randomized controlled trial of an algorithm based upon the WHO ladder demonstrated that a standardized approach to cancer pain management using the algorithm provided more effective analgesia than routine oncology care. 10

There are multiple treatment options for patients with painful bone metastases which include, surgical management, external beam radiation therapy, systemic therapy and radionuclide therapy ${ }^{11}$. Bone metastases are the most common indication for palliative radiotherapy. ${ }^{12}$ There are multiple randomized trials which have compared between single and multiple fraction radiotherapy, in order to assess the pain relief. It has been found that most of the response in terms of pain relief occurs 4-6 weeks after radiation. A sustained pain relief is seen in approximately $55-65 \%$ of patients. ${ }^{12}$

The response to treatment depends on a large number of factors $^{13}$ like

1. Sex.

2. Primary site.

3. Histology.

4. Performance status.
5. Type of lesion (Osteolytic vs. Osteoblastic).

6. Location of the metastases (weight-bearing vs. non-weight-bearing).

7. Extent of disease.

8. Number of painful sites.

9. Level of pain prior to treatment.

The effectiveness of the treatment also depends on the goal: palliation of pain, prevention of pathologic fracture, avoidance of future treatments, or local control of the disease.

Local-field external-beam radiation therapy is typically used for palliation of a few discrete areas of painful metastases. ${ }^{12}$ Various doses and fractionations have been tested in randomized trials, and results from most trials demonstrated that a large single dose and more fractionated radiation provided similar symptomatic control. There have been various studies in the past comparing different fractionation schedules which varying from $40 \mathrm{GY}$ to $8 \mathrm{GY}$. However, the most commonly used fractionation are 30 GY/10\#, 20GY/5\#, and 8GY/1 \#. There are no level one evidence favouring any of the above-mentioned regimen. Nevertheless, in a high volume centers long fractionation schedules will increase the machine time and there by more patient waiting. It will not only affect the palliative treatments but also influence the starting time in radical cases. In this scenario many high volume centers invariably opts for the shorter versions of radiotherapy in palliative cases, our study is to retrospectively analyse the case sheets to assess the effectiveness of shorter versions of radiotherapy in palliative scenarios.

\section{Aim of The Study}

This study is to evaluate the efficacy of external beam radiation therapy in achieving pain relief for patients with painful bony metastases. The two radiation fractionation schedules compared in this study are multiple fraction $(20$ Gy/ 5 fractions) and single fraction treatment (800 cGy single fraction).

In addition, the change in analgesic requirement between the two arms has also been assessed in this study.

\section{METHODS}

This study is a retrospective cohort study of Case records of all patients who are reported to MCH Kottayam with diagnosis of Bone metastasis between the duration of August 2010 to January 2015 and are treated with radiotherapy dose of either $20 \mathrm{GY} / 5 \#$ or 8 gy/1\# were collected and data about the response to treatment was retrieved. Data of 150 patients were eligible for the study and was segregated into two groups based on the dose fractionation regimen. Patients were excluded if no retrievable data was available from the case records.

\begin{tabular}{|c|c|c|}
\hline Dose of Radiotherapy & 20 GY/5\# & 8GY/1\# \\
\hline Total number of patients & 96 & 84 \\
\hline $\begin{array}{c}\text { Total number of patients } \\
\text { included in the analysis }\end{array}$ & 75 & 75 \\
\hline
\end{tabular}

\section{Inclusion Criteria}

1. Age 18 years or older.

2. Patients must have signed an approved informed consent. 
3. Patients with a proven primary (solid tumour) malignancy presenting with painful bony metastases and treated with palliative radiation with either $20 \mathrm{GY} / 5 \#$ OR 8 GY/1\#

\section{Exclusion Criteria}

1. Patients with a diagnosis of multiple myeloma, lymphoma or haematological malignancies.

2. Patients with painful bony metastases in more than 5 sites.

3. Patients with pathological fracture, spinal cord compression.

4. Patients treated with other dose fractionation schedules.

\section{Duration of Study}

Patient's data were analysed over a period of 4 months after obtaining ethical clearance.

\section{Sample Size}

Retrospective Cohort Study.

\section{Statistical Analysis}

Data was entered in Microsoft Excel \& analysis was done. SPSS software version 15 was used for analysis. The pain score data in two group (20 Gy/5\# and $8 \mathrm{~Gy} / 1 \#$ ) per week is compared with student $t$ test. Qualitative variables are expressed in percentage or proportion and association of reduction in pain from baseline to observational priod of 12 weeks analysed using chi-square test.

\section{RESULTS}

The patients were evaluated. Patients who were irregularly followed up or with missing data from case sheets were also excluded from the study. After careful evaluation, 75 patients each were chosen in the two groups and each patient compared with the other group to evaluate results.

In this study 150 patients satisfied the criteria of patient selection from January 2012 to December 2015.

\section{Study Procedure}

- Patients meeting the inclusion and exclusion criteria will be included in the study.

- The patients who received the above mentioned 2 different fractionations will be compared and analysed.

- The baseline pain score and the patient reported pain score which has been recorded according to the institutional protocol in the master case sheet was retrieved and analysed.

- The data on prescription of analgesic according to the WHO pain ladder which was be recorded in the Master case sheet will be evaluated.

- Retrospective comparison of these factors between the two arms will be used to determine the better treatment protocol for pain control.

All patients accrued in this study will be planned for external beam radiation using the conventional simulator housed in the Department of Radiotherapy at our center. The treatment fields were defined following x-ray imaging of the involved region on the $\mathrm{x}$-ray simulator. For vertebral metastases, one vertebra above and below the involved vertebra would be included in the radiation portal, the lateral extent of the field would encompass the transverse process of the vertebra bilaterally. For bone metastases at other sites, a minimum of $2 \mathrm{~cm}$ margin would be given around the radiologically visible lesion.

The number of treatment fields were decided by the depth of the region of interest. Vertebral lesions were treated using a single on field technique, whereas other sites would be treated with opposing anterior and posterior beams. All patients in this study will be treated with the Theratron 780C Telecobalt therapy unit housed in the Department of Radiotherapy, at our institute.

As per institutional protocol all patients will be given bisphosphonate therapy after ensuring that the renal function and the serum calcium levels were within normal limits. All patients were sent to physical medicine department so as to devise braces/ support measures so as to enable early rehabilitation and ambulation. All patients were started on analgesics based on their pain score keeping in tune with the WHO analgesic ladder.

\section{Assessment Tools}

To assess the pain, Universal pain assessment tool was adopted.

\begin{tabular}{|c|c|}
\hline Step & Drugs \\
\hline 1 & Paracetamol, NSAIDS (and adjuvants if needed) \\
\hline 2 & $\begin{array}{c}\text { Mild Acting Opioids + Step l Non-Opioids (and } \\
\text { adjuvants if needed) Mild Acting Opioids: Codeine, } \\
\text { Dihydrocodeine, Dextropropoxyphene }\end{array}$ \\
\hline 3 & $\begin{array}{c}\text { Stronger Opioids + Step L Non-Opioids (and adjuvants } \\
\text { if needed) Stronger Opioids; Morphine, Diamorphine, } \\
\text { Fentanyl, Hydromorphone }\end{array}$ \\
\hline \multicolumn{2}{|c|}{ Table 1. WHO Analgesic Ladder } \\
\hline
\end{tabular}

\section{Adjuvant Drugs}

Tricyclic Antidepressants, anti convulsants, Steroids.

\section{Definitions}

Case

Patients with a proven primary (Solid tumour) malignancy presenting with painful bony metastases.

\section{Response}

Decrease in pain score by at least 2 points.

\section{No Response}

If the pain score remains at the pre-treatment level.

\section{Progression}

Worsening of pain score after completion of treatment.

\begin{tabular}{|c|c|}
\hline $\begin{array}{c}\text { Analgesic } \\
\text { Response }\end{array}$ & $\begin{array}{c}\text { \% Decrease in Analgesic Requirement } \\
\text { (Dose in mg) }\end{array}$ \\
\hline Mild & At Least 25\% \\
\hline Moderate & $25 \%-50 \%$ \\
\hline Good & $50 \%-75 \%$ \\
\hline Excellent & $75 \%-100 \%$ \\
\hline \multicolumn{2}{|c|}{ Table 2. Analgesic Response } \\
\hline
\end{tabular}




\section{Primary Site}

Lung cancer cases accounted for $50 \%$ of the patients in this study $20 \%$ of the patients in this study had breast as the primary site of disease.

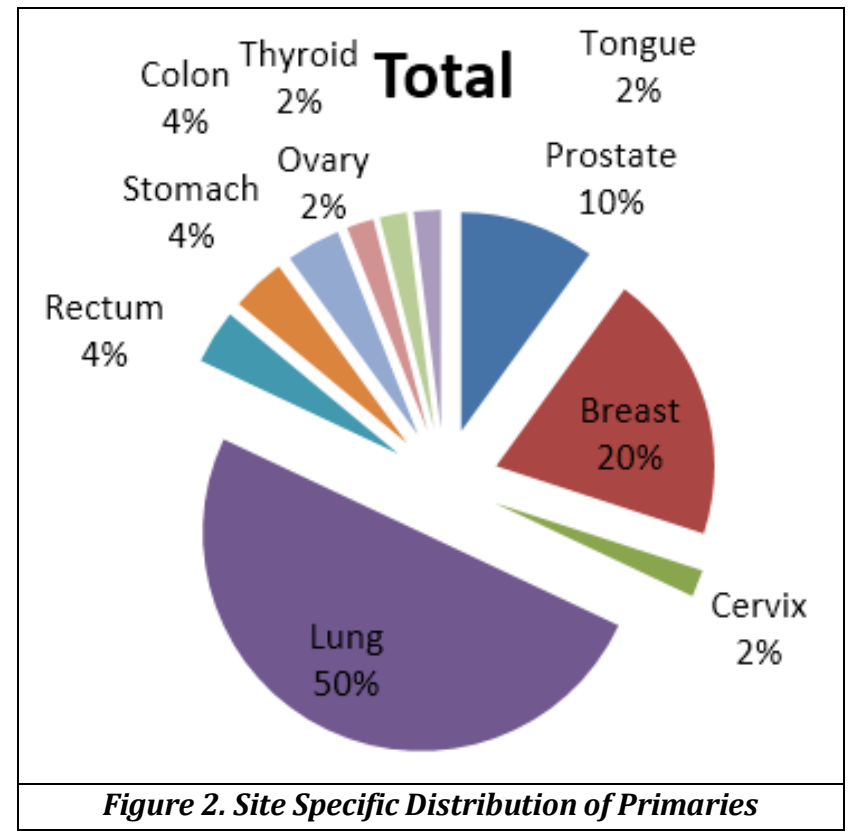

\section{Site of Bone Metastases}

In this study bone metastases were recorded in the pelvis, vertebra, rib \& femur. $74 \%$ of bone metastases in this study occurred in the vertebra.

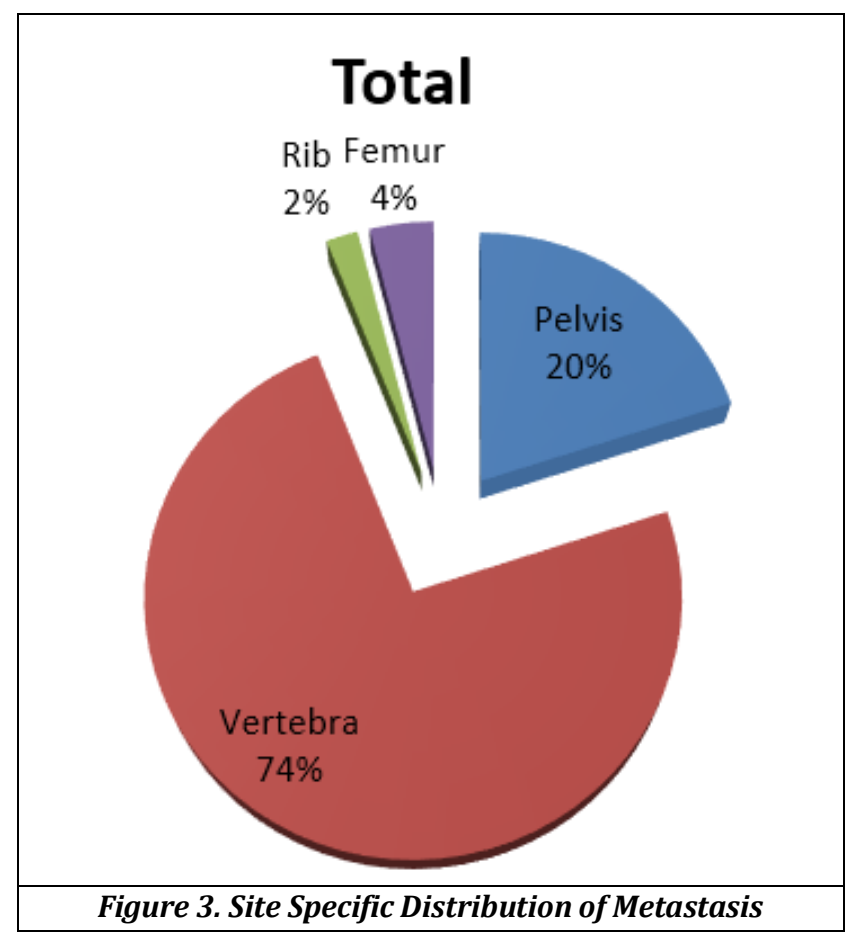

As vertebral metastases were found to account for majority of the cases, a subset analysis showed that lumbar vertebra accounted for $48.6 \%$ of all vertebral metastases. Sites of bone metastases were further subdivided into weight-bearing sites Include vertebra in weight bearing site. (i.e., pelvis, femur, tibia, sacrum, and/or sacroiliac joints) or non-weight-bearing sites.

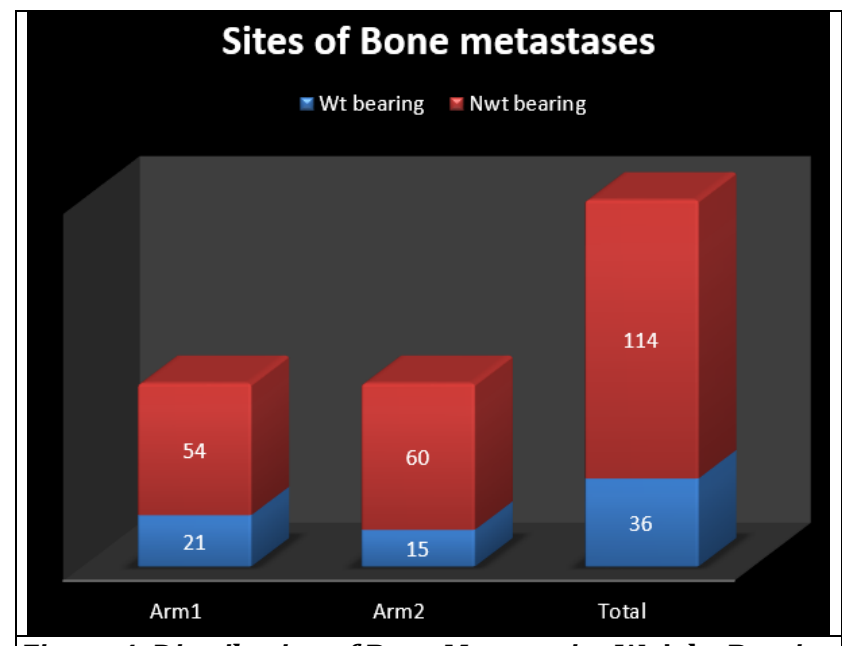

Figure 4. Distribution of Bone Metastasis- Weight Bearing $v / s$ Non-Weight Bearing

$76 \%$ of patients presented with bone metastases to nonweight bearing sites. No new bone metastases were recorded in any of the patients during the period of this study.

\section{Pain Score}

Pain was assessed using the universal pain assessment tool. Patients graded pain from zero (No pain) to ten (Worst possible pain).

Pain was graded according to the Pain Score as follows

\begin{tabular}{|c|c|}
\hline Pain Grade & Pain Score \\
\hline Mild & $1-2$ \\
\hline Moderate & $3-6$ \\
\hline Severe & $7-10$ \\
\hline \multicolumn{2}{|c|}{ Table 3. Pain Grade } \\
\hline
\end{tabular}

Pain score was assessed at presentation. The mean pain score at presentation was 6.82. The pain score at presentation ranged from five to nine.

Arm 1 had 30 patients with moderate pain and 45 patients with severe pain. Arm 2 had 36 patients with moderate pain and 39 patients with severe pain.

\section{Pain Score at First Follow Up (2nd Week)}

The mean pain score at first follow up was 6.78. The pain score at first follow up ranged from four to nine. There was no statistically significant difference in pain scores at first follow up between the two arms. $p$ value $=0.438$

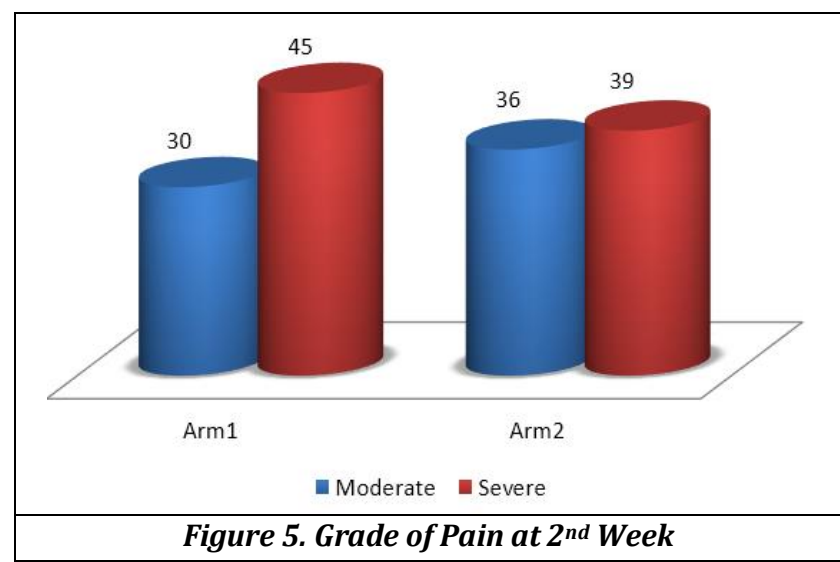


From the figure depicted above it is clear that the distribution of patients with moderate and severe pain remains the same as at presentation.

\section{Pain Score at Second Follow Up (3rd Week)}

The mean pain score at second follow up was 6.62 . The pain score at second follow up ranged from four to nine. There was no statistically significant difference in pain scores at second follow up between the two arms. $p$ value $=0.15$. The number of patients with moderate and severe pain remain the same in Arm 1. In Arm 2 the number of patients with moderate pain has increased from 36 to 42 , thereby decreasing the number of patients with severe pain from 39 to 33 .

The mean pain score at third follow up was 5.88. The pain score at third follow up ranged from one to nine. There was no statistically significant difference in pain scores at third follow up between the two arms. $p$ value $=0.738$. In each arm 3 patient reported mild pain. Compared to the second follow up the number of patients reporting severe pain has decreased in both the arms. A greater proportion of patients report moderate pain in both the arm.

The mean pain score at fourth follow up was 4.48. The pain score at fourth follow up ranged from one to one to eight. There was no statistically significant difference in pain scores at fourth follow up between the two arms. $\mathrm{p}$ value $=$ 0.738. At fourth follow up, it can be noted that the number of patients reporting severe pain has further decreased in both arms. At the same time the number of patients reporting mild pain has increased in both arms. The number of patients reporting moderate pain has remained more or less the same in both the arms.

The mean pain score at fifth follow up was 3.69. The pain score at fifth follow up ranged from one to one to nine. There was no statistically significant difference in pain scores at fifth follow up between the two arms. $p$ value not significant. 3 patient in arm 2 reports no pain. A steady decrease in number of patients reporting severe pain is noted in both arms. The proportion of patients reporting mild pain is increasing in both arms, whereas number of patients reporting moderate pain has remained more or less constant in both arms.

The mean pain score at sixth follow up was 3.08. The pain score at sixth follow up ranged from one to one to nine. There was no statistically significant difference in pain scores at sixth follow up between the two arms. $p$ value =NS. Both the arms have 4 patient each reporting no pain. The trend observed previously is continued here, with decreasing number of patients reporting severe pain in both arms. The proportion of patients reporting mild pain has been consistently increasing in both arms, with a more profound increase in arm 2 . The mean pain score at seventh follow up was 3.22. The pain score at seventh follow up ranged from one to one to nine. There was no statistically significant difference in pain scores at seventh follow up between the two arms.
The number of patients in arm 1 reporting no pain has increased from 3 to 12, whereas no such increase has been noted in arm 2. The number of patients reporting mild pain has remained fairly the same in both arms. No further decrease in the number of patients reporting severe pain was noticed.

\begin{tabular}{|c|c|c|c|c|c|c|}
\hline 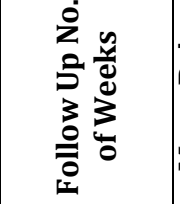 & 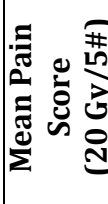 & 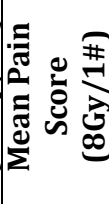 & 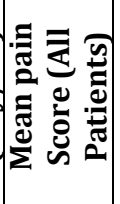 & 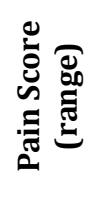 & 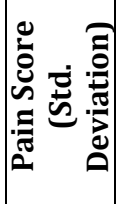 & 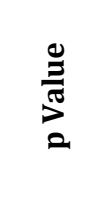 \\
\hline $\begin{array}{c}\text { At } \\
\text { Presentation } \\
\text { (baseline) }\end{array}$ & 6.92 & 6.72 & 6.82 & $5-9$ & 1.224 & \\
\hline $2^{\text {nd }}$ Week & 6.92 & 6.64 & 6.78 & $4-9$ & 1.282 & 0.438 \\
\hline 3rd Week & 6.88 & 6.36 & 6.62 & $4-9$ & 1.260 & 0.155 \\
\hline $4^{\text {th }}$ Week & 5.96 & 5.8 & 5.88 & $1-9$ & 1.814 & 0.738 \\
\hline $6^{\text {th } W e e k}$ & 4.92 & 4.04 & 4.48 & $1-8$ & 2.150 & 0.117 \\
\hline $8^{\text {th }}$ Week & 3.76 & 3.63 & 3.69 & $1-9$ & 2.153 & 0.683 \\
\hline 10th Week & 3.83 & 2.85 & 3.08 & $1-9$ & 2.211 & 0.429 \\
\hline $12^{\text {th }}$ Week & 3.71 & 2.79 & 3.22 & $1-9$ & 2.344 & 0.141 \\
\hline \multicolumn{7}{|c|}{ Table 4. Summary of Pain Scores } \\
\hline
\end{tabular}

The difference in mean pain score at 12 weeks from baseline mean pain score (At week 0 ) has been evaluated by Chi Square test. There is a statistically significant reduction in pain with radiation from baseline (wk. 0) during the period of study (wk 12) with chi square statistics 7.9093 and a significant $\mathrm{p}$ value, $\mathrm{p}=0.004918$. But the reduction in mean pain scores between the $20 \mathrm{~Gy} / 5 \#$ and $8 \mathrm{~Gy} / 1 \#$ are did not show any statistical difference across each week throughout this study. The variables between the 2 groups in each week were analysed by Chi-square test with $\mathrm{p}=0.5678$ shows that the reduction in pain with radiation is observed similar between the 2 groups of radiation fractionation used in this study of $20 \mathrm{~Gy} / 5 \#$ and $8 \mathrm{~Gy} / 1 \#$ and the difference is statistically not significant.

The graph above shows that a steep decrease in mean pain score is noted from the $4^{\text {th }}$ week onwards i.e. from 5.88 to 4.48 at the $6^{\text {th }}$ week. This decrease is mean pain score is noted up to the $10^{\text {th }}$ week, after which a non-significant increase in mean pain score is noted at the $12^{\text {th }}$ week.

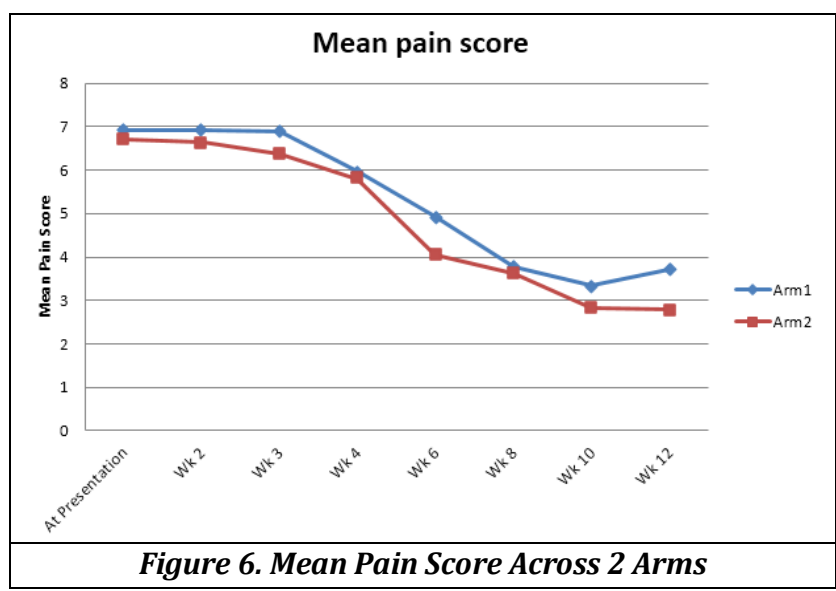




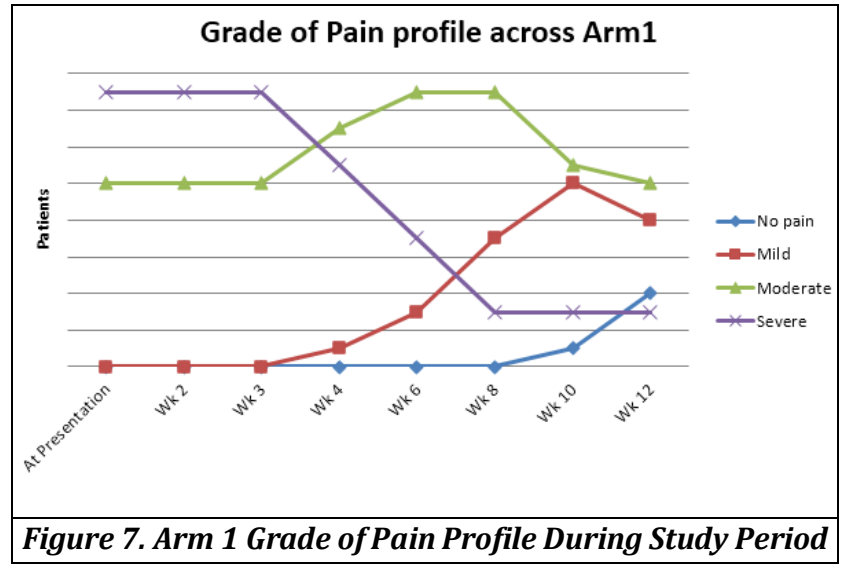

The line graph in figure 7 shows that initially all patients in Arm 1 reported severe and moderate pain. There was a sharp decline in patients reporting severe pain from week 3 onwards, this decrease continued up to week 8 . The number of patients reporting moderate pain increased from week 3 and stabilized from week 6-8, thereafter a steady decrease is noted. Patients reporting mild pain increased from week 3 onwards, reaching a maximum at week 10 . The number of patients reporting mild pain started increasing from week 8 onwards, peaking at week 12 .

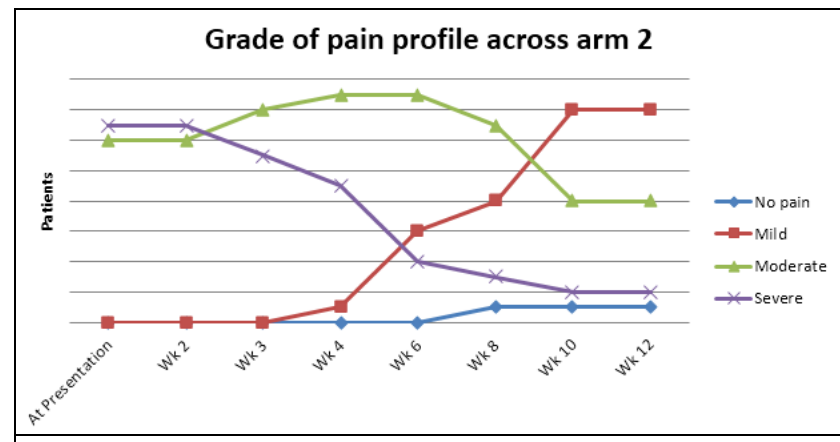

Figure 8. Arm 2 Grade of Pain Profile During Study Period

\section{Pain Response}

The $\mathrm{p}$ value for pain response was 0.695 , there by proving that there was no statistically significant difference in pain response between both the arms.

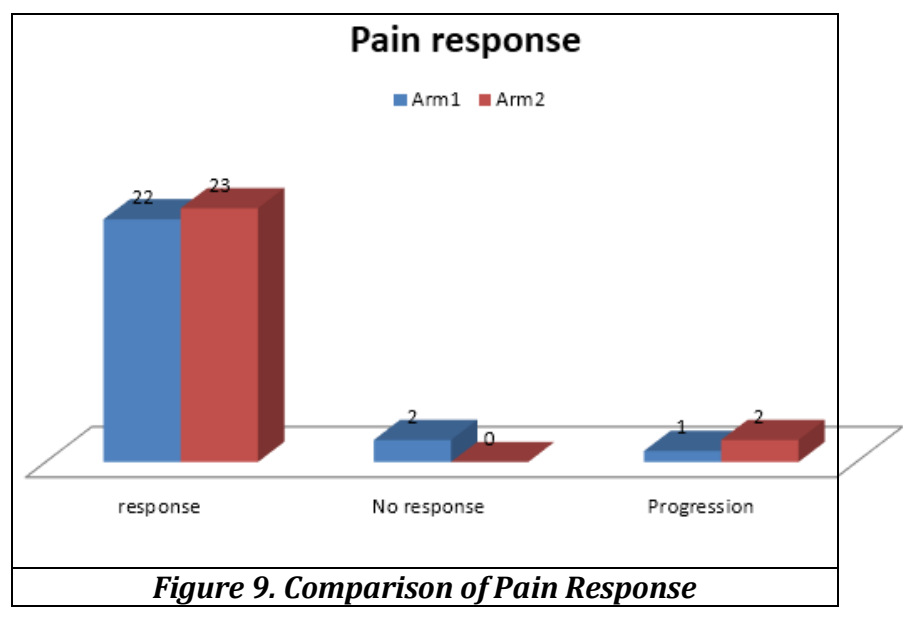

The bar graph shows an equitable distribution between the 2 arms with respect to number of patients achieving a pain response, no pain response $\&$ progression of pain.

\section{Analgesic Step}

At presentation all patients were started on Step 1 of the WHO analgesic ladder. At presentation all patients were on either step 2 or 3 . By week 12 it is observed that more than $50 \%$ of patients were on No or step 1 analgesics. This observation was not statistically different between 2 arms.
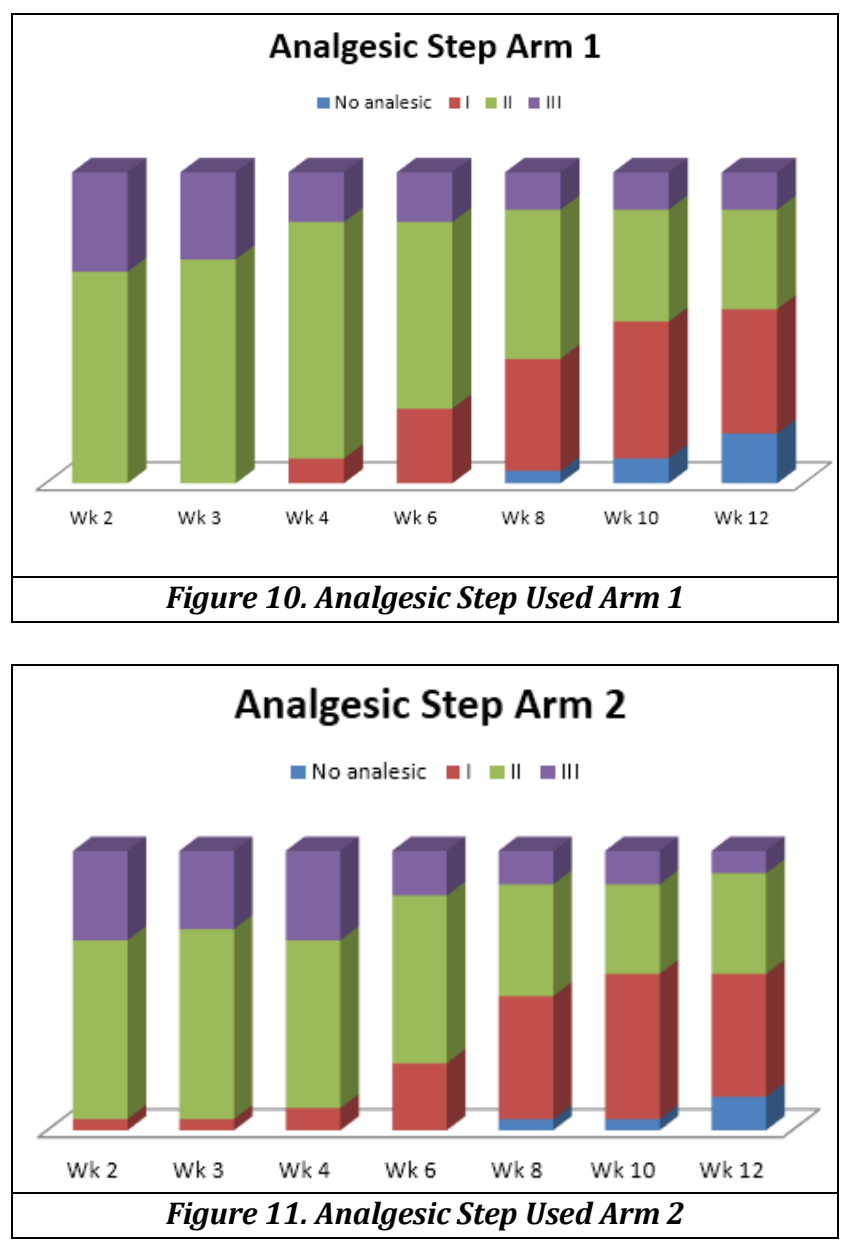

\section{DISCUSSION}

Various randomized trials were conducted for comparing Radiation fractionation and pain relief. In RTOG $9714^{14}$ study it is found that external beam radiation therapy was effective at palliating pain from bone metastases, with complete or partial improvement in pain observed at 3 months after randomization in $66 \%$ (375 patients) of the 573 patients. At 3 months of follow-up. Similar to the results of our study, this study found no difference between the response of patients in the arm receiving $30 \mathrm{GY}$ in 10 treatment fractions and in the arm receiving $8 \mathrm{GY}$ in a single treatment fraction, in terms of pain relief, narcotic relief, or pathologic fracture incidence, regardless of stratification used in the analysis. Treatment was well tolerated with few adverse effects.

The complete response rate in the RTOG 9714 trial was $16 \%$, substantially lower than the previous RTOG 740215 study. The reasons for this difference may include the assessment method used and the severity of pain or extent of disease. For the RTOG 7402 trial, physicians scored pain with a four-point scale, whereas patients in our study scored pain by use of a more sensitive 10-point scale in the Brief Pain Inventory. In addition, the patients treated in this study is different from that treated 25 or more years ago. Although there were few systemic therapy options during the RTOG 
7402 trial, second-, third-, and fourth line chemotherapy options are currently available for breast cancer. In addition, multiple hormonal manipulations are available for the treatment of both breast and prostate cancer, and bisphosphonates are used in many of these patients.

Pain control is better understood, with much more emphasis on adequate pain management now than 25 years ago. Thus, the patients who are referred for palliative radiation therapy now may have more widespread disease that has become resistant to other therapies, as reflected in this study by the severity of pain scores $(72 \%$ of the patients in this study had severe pain at study entry) and the Karnofsky performance status scores (nearly 25\% of the patients in this study had Karnofsky performance status scores of 60 or lower, and more than $75 \%$ had Karnofsky performance status scores of 80 or lower). The quality-of-life assessments also show that the patients in this study have many symptoms. Although this group of patients was ill with moderate-to-severe pain before treatment with radiation therapy, a substantial proportion had improvement in pain 3 months after treatment, and nearly one-third no longer required narcotic pain medication. The results may have been better if patients were treated earlier in the course of their disease, since previous studies have shown that patients with moderate pain are more likely to respond to treatment than those with severe pain ${ }^{1}$.

The only difference in outcomes between the two arms was the rate of re-treatment, with substantially more patients in the 8-Gy arm receiving retreatment than in the 30 Gy arm. This observation may be an indication that the 8-Gy treatment is less effective than the longer course of $30 \mathrm{~Gy}$ in 10 treatment fractions. However, rates of pain relief, narcotic use, and pathologic fracture incidence were equivalent in the two treatment arms. There may be other factors involved in the decision to re-treat a patient, such as potential physician bias. There may be more willingness to give another treatment after a single-dose treatment than after a higherdose treatment, especially retreating areas adjacent to sensitive critical normal structures (such as spinal cord, bowels, or lungs). There may be less willingness to give another treatment after a treatment of 30 Gy in 10 fractions because of the higher acute toxicity associated with that regimen. Even if there is a real increase in the need for retreatment among patients receiving a single-dose treatment, this problem may be counter balanced by the reduced rate of acute toxicity in these patients.

A consensus statement from the Second Workshop on Palliative Radiotherapy and Symptom Control in 200016 confirmed the efficacy of radiation therapy, even with a single treatment, in palliating painful bone metastases. There is increasing evidence that a single 8-Gy dose provides pain relief equivalent to longer courses of palliative treatment, although the short course of treatment is associated with a higher rate of retreatment.

This study has several limitations. This study included only patients with metastases from primary breast or prostate cancers to allow an adequate follow-up period to assess response and toxicity, because these patients tend to survive longer than patients with bone metastases from other primary sites. However, the outcomes may be different for patients with bone metastases from another primary site. A second limitation of the study involves completion of the assessment tool. The Brief Pain Inventory was completed by only $573(67.8 \%)$ of the 845 patients at the 3-month assessment point. As would be expected in this group of patients, 160 of the 845 patients had died or were too ill to complete the form at 3 months. Thus, the Brief Pain Inventory was completed by $573(83.6 \%)$ of the 685 patients who were alive and able to complete the form.

\section{CONCLUSIONS}

$20 \mathrm{~Gy} / 5$ fractions and $8 \mathrm{~Gy} /$ single fraction schedules of radiotherapy were found to be similar in terms of rates of pain relief, narcotic use, and pathologic fracture. In terms of acute control of patient symptoms of bone pain, either of the two schedules may be used without compromising outcome.

\section{REFERENCES}

[1] Coleman RE. Skeletal complications of malignancy. Cancer 1997;80(8 Suppl):1588-94.

[2] Asdourian PL, Weidenbaum M, DeWald RL, et al. The pattern of vertebral involvement in metastatic vertebral breast cancer. Clinical Orthopaedics and Related Research 1990;(250):164-70.

[3] Halperin EC, Wazer DE, Brady LW, et al. Perez and Brady's Principles and practice of radiation oncology. $6^{\text {th }}$ edn. Philadelphia, PA, USA: Lippincott Williams \& Wilkins 2013.

[4] Macedo F, Ladeira K, Pinho F, et al. Bone metastases: an overview. Oncology Reviews 2017;11(1):321.

[5] Goblirsch MJ, Zwolak PP, Clohisy DR. Biology of bone cancer pain. Clinical Cancer Research: an Official Journal of the American Association for Cancer Research 2006;12(20 Pt 2):6231s-5s.

[6] Agarwal MG, Nayak P. Management of skeletal metastases: an orthopaedic surgeon's guide. Indian Journal of Orthopaedics 2015;49(1):83-100.

[7] Daldrup-Link HE, Franzius C, Link TM, et al. Wholebody MR imaging for detection of bone metastases in children and young adults: comparison with skeletal scintigraphy and FDG PET. American Journal of Roentgenology 2001;177(1):229-36.

[8] Cleeland CS, Gonin R, Hatfield AK, et al. Pain and its treatment in outpatients with metastatic cancer. The New England Journal of Medicine 1994;330(9):592-6.

[9] Cleeland CS. The measurement of pain from metastatic bone disease: capturing the patient's experience. Clinical Cancer Research: an Official Journal of the American Association for Cancer Research 2006;12(20 Pt 2):6236s-42s.

[10] Nersesyan H, Slavin KV. Current approach to cancer pain management: availability and implications of different treatment options. Therapeutics and Clinical Risk Management 2007;3(3):381-400.

[11] Nielsen OS, Munro AJ, Tannock IF. Bone metastases: pathophysiology and management policy. Journal of Clinical Oncology: Official Journal of the American Society of Clinical Oncology 1991;9(3):509-24.

[12] De Felice F, Piccioli A, Musio D, et al. The role of radiation therapy in bone metastases management. Oncotarget 2017;8(15):25691-9. 
[13] Choi C, Seong J. Predictive factors of palliative radiotherapy response and survival in patients with spinal metastases from hepatocellular carcinoma. Gut and Liver 2015;9(1):94-102.

[14] Hartsell WF, Scott CB, Bruner DW, et al. Randomized trial of short- versus long-course radiotherapy for palliation of painful bone metastases. Journal of the National Cancer Institute 2005;97(11):798-804.
[15] Salazar OM, Rubin P, Hendrickson FR, et al. Singledose half-body irradiation for the palliation of multiple bone metastases from solid tumours: a preliminary report. International Journal of Radiation Oncology, Biology, Physics 1981;7(6):773-81.

[16] Hoskin PJ, Yarnold JR, Roos DR, et al. Radiotherapy for bone metastases. Clinical Oncology 2001;13(2):88-90. 